\title{
Small-Scale $\frac{1}{4} \mathrm{keV}$ Fluctuations Due to the Local Hot Bubble
}

\author{
K.D. Kuntz ${ }^{1}$, S.L. Snowden ${ }^{2}$, and R.S. Warwick ${ }^{3}$ \\ 1 University of Maryland, College Park, Maryland, 20742, USA \\ 2 USRA/GSFC, Greenbelt, Maryland, 20771, USA \\ 3 University of Leicester, University Road, Leicester LE1 7RH, United Kingdom
}

\begin{abstract}
To determine fluctuations in the $\frac{1}{4} \mathrm{keV}$ emission due to the LHB, without contamination due to sources within the galactic disk, the Galactic halo or the extragalactic background, we have formed a mosaic of PSPC observations towards the Hyades, a direction where distant emission is effectively blocked by a Galactic column of many optical depths. After removing the point sources from this image, we find the $\frac{1}{4} \mathrm{keV}$ emission to be smooth on scales from $6^{\prime}$ to a few degrees.
\end{abstract}

\section{Introduction}

Barber, Warwick, \& Snowden (1995) discovered small-scale fluctuations in the $\frac{1}{4} \mathrm{keV}$ emission in the UK medium deep survey region, high galactic latitude field. These fluctuations have amplitudes of $10 \%$ to $20 \%$ of the total $\frac{1}{4} \mathrm{keV}$ background, and have scale-sizes of about $20^{\prime}$ (Warwick et al, 1997). To characterize these fluctuations, and to isolate the component of the $\frac{1}{4} \mathrm{keV}$ background from which they arise, it is necessary to measure the spectrum of fluctuations from all of the "foreground" background components. We present here a measure of the small-scale fluctuations due to the Local Hot Bubble (LHB).

To determine the contribution due to the LHB, without contributions from galactic halo or extragalactic components, it is necessary to observe the LHB in the direction of background masses of neutral hydrogen optically thick to $\frac{1}{4} \mathrm{keV} \mathrm{X}$-rays. The Hyades cluster, which contains many overlapping PSPC fields, lies in the direction of the Taurus-Auriga molecular clouds. The $\frac{1}{4} \mathrm{keV}$ opacity $\left(N_{H} \times \sigma_{e f f}\right)$ of the Taurus-Auriga clouds in the direction of the PSPC observations is greater than $\sim 5$, effectively blocking extragalactic and halo $\frac{1}{4} \mathrm{keV}$ emission.

\section{Data}

We have mosaicked all of the overlapping PSPC pointed observations towards the Hyades cluster. Individual exposures were reduced using the ESAS software (Snowden 1995) in the manner described by Kuntz, Snowden, \& Verter 


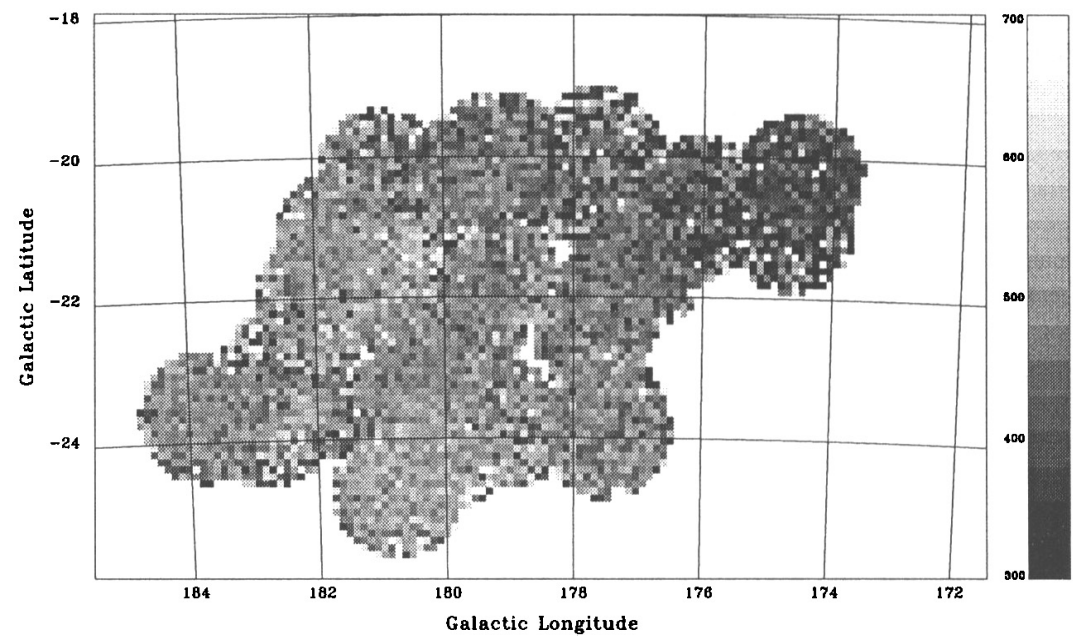

Fig. 1. $\frac{1}{4} \mathrm{keV}$ (band $\mathrm{R} 1 \mathrm{~L}+\mathrm{R} 2$ ) image of the Hyades cluster after point source removal. The pixel size is $6^{\prime} \times 6^{\prime}$, the units are $10^{-6}$ counts $\mathrm{s}^{-1} \operatorname{arcmin}^{-2}$. The pixel-to-pixel variation is indicative of the statistics; regions with high pixel-to-pixel variation have low exposure. The "bright" spots at $(\ell, b) \sim\left(178^{\circ},-21^{\circ}\right)$ and $(\ell, b) \sim\left(178.5^{\circ},-23^{\circ}\right)$ are "holes" in the mosaic.

(1997) and references therein. Point sources were removed from the individual exposures which were then mosaicked together in the manner described by Snowden (1995). The mosaic covers nearly $35 \mathrm{deg}^{2}$, contains exposures from $<1 \mathrm{ks}$ to $\sim 32 \mathrm{ks}$, and is shown in Figure 1.

\section{Analysis}

Although the $\frac{1}{4} \mathrm{keV}$ image shows some large scale structure, there is little structure on scales smaller than a degree. We have employed two methods for characterizing the smoothness on these scales; the autocorrelation function $(\mathrm{ACF})$ and the $\sigma$-normalized distribution.

We have calculated the autocorrelation function for the point-sourceremoved image with $6^{\prime} \times 6^{\prime}$ pixels. As can be seen in Figure 2, the overall trend of the $\mathrm{ACF}$ is consistent with the large scale structure, i.e., a peak at about $(\ell, b)=\left(180.5^{\circ},-21^{\circ}\right)$, and lower emission regions at $\left(175^{\circ},-21^{\circ}\right)$ and $\left(184^{\circ},-23^{\circ}\right)$. There is power on scales smaller than a few degrees, but the slope between 0 and $2^{\circ}$ implies that it is due to large-scale structure rather than to small-scale fluctuations.

The ACF is not an effective tool for scales smaller than a few times the pixel size. To more carefully test the existence of fluctuations at smaller scales, we model the pixel-to-pixel variations. The comparison at different pixel sizes places limits upon the non-statistical fluctuations at various scales. 


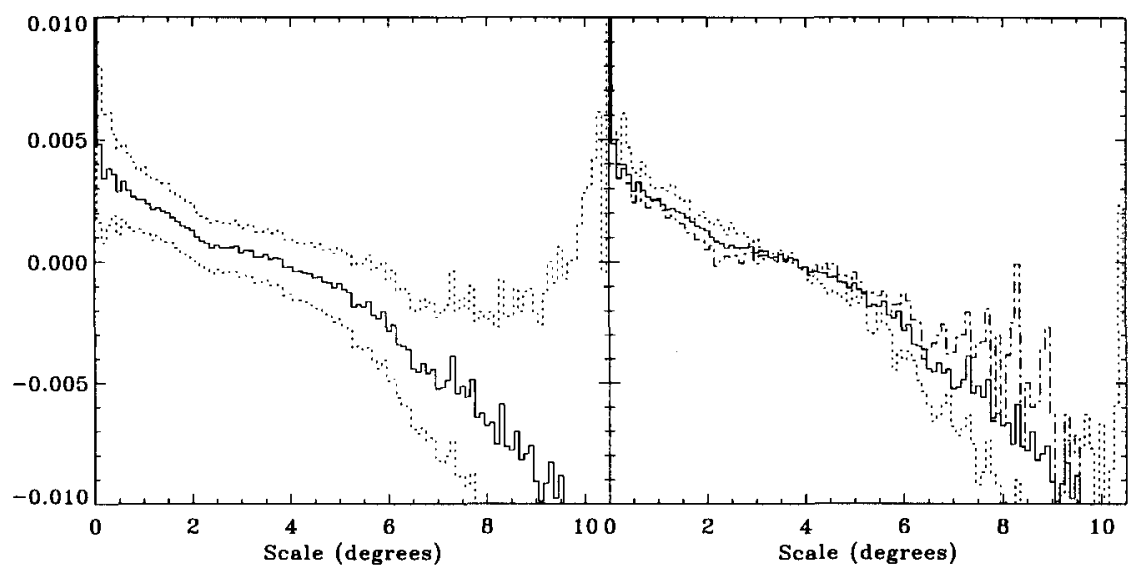

Fig. 2. Left: The ACF for the mosaic in the band R1L+R2. The dotted lines show the formally propagated uncertainty. Right: The ACF for the band R1L+R2 (solid), compared to the ACF for bands R1L (dashed) and R2 (dot-dashed).

We define a $\sigma$-normalized distribution for an image with a count rate $\rho(x, y)$ and uncertainty $\sigma(x, y)$ to be the distribution of the quantity

$$
\frac{\rho(x, y)-\overline{\rho(x, y)}}{\sigma(x, y)}
$$

where $\overline{\rho(x, y)}$ is the mean of the eight pixels adjacent to position $(x, y)$. The $\sigma$-normalized distribution is shown in Figure 3.

To model the expected distribution we have taken the exposure map and multiplied it by the mean count rate. To this image is added the same modeled non-cosmic background (scattered solar X-rays, long-term X-ray enhancement, etc.) used in the reduction. (This is a model "raw" image.) We then create an image where an individual pixel value is randomly drawn from a Poisson distribution whose mean is the model "raw" count at that pixel. From this image is subtracted the non-cosmic background; the result is divided by the exposure time. Point sources thought to exist below the detection threshold can be then added to produce a model image, however we find that the addition of only a very small flux in point sources can be tolerated before the model diverges greatly from the data.

The data and the model do not agree in a statistical sense, but, we can test the effect of small-scale fluctuations. The right-hand panel of Figure 3 shows that the addition of $2.5 \%$ fluctuations (pixel-to-pixel variations) on $6^{\prime}$ scales are clearly incompatible with the data. The difference becomes greater with pixel size. More careful modeling of the $20^{\prime}$ fluctuations such as those found by Barber, Warwick, \& Snowden (1995), must be done before their existence in the LHB can be confidently excluded. 


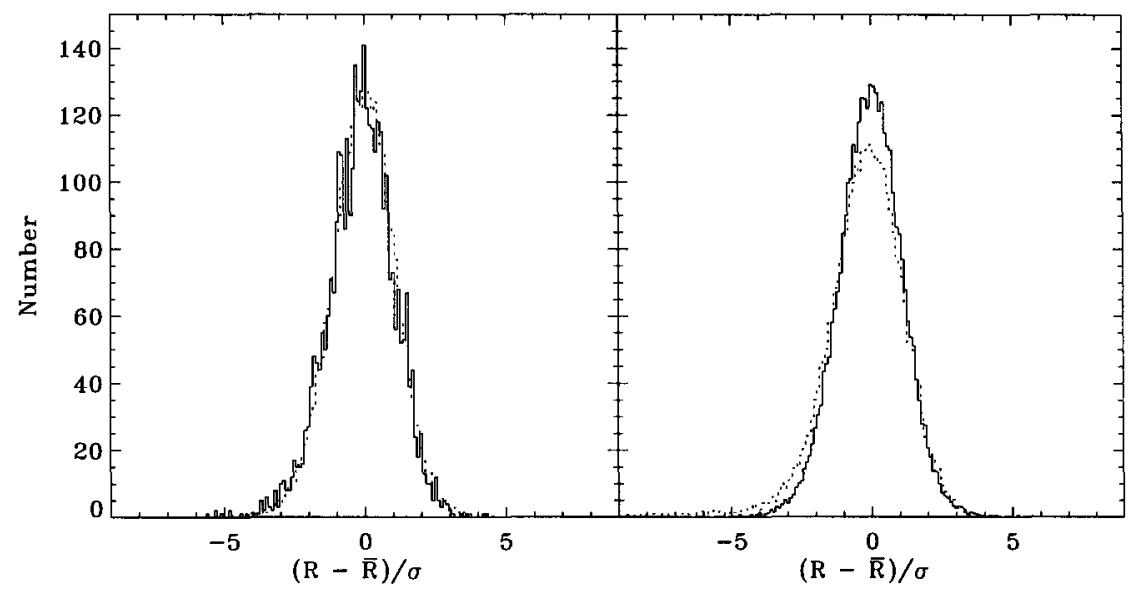

Fig. 3. Left: The $\sigma$-normalized distribution for the mosaic in the band R1L+R2 (solid line). The pixel size was $6^{\prime} \times 6^{\prime}$. The model distribution (dotted line). Right: The model distribution (solid line). The model distribution with an additional $2.5 \%$ fluctuation (dotted line). Model distributions are the mean of 10 trials.

\section{Discussion}

We have found the $\frac{1}{4} \mathrm{keV}$ emission from the LHB to be smooth on scales from $6^{\prime}$ to $\sim 1^{\circ}, 0.13 \mathrm{pc}$ to $\sim 1.3 \mathrm{pc}$ at the edge of the LHB (d $\sim 70 \mathrm{pc}$ ). If fluctuations were caused by a change in the path-length to the LHB wall; a $0.13 \mathrm{pc}$ wide fluctuation could have a depth of no more than $1.8 \mathrm{pc}$. Wider fluctuations will be more tightly constrained. Thus, there are no low-columndensity "tunnels" on this scale, as might be expected were the LHB optical depth bounded. This result may also place constraints on the degree to which edge brightening may contribute to the LHB emission, as well as constraints upon the small scale structure of $\mathrm{H}$ I embedded in the LHB. We continue this work to more tightly constrain small-scale fluctuations.

\section{References}

Barber, C., Warwick, R. S., Snowden, S. L. (1995): Röntgenstrahlung from the Universe, eds., Zimmermann, H.U., Trümper, J.E., \& Yorke, H., 319.

Kuntz, K.D., Snowden, S.L., Verter, F. (1997): ApJ, in press

Snowden, S. L. (1995): Cookbook for Analysis Procedures for ROSAT XRT/PSPC Observations of Extended Objects and the Diffuse Background.

Warwick, R., Hutchinson, I, Willingale, R., Kuntz, K., \& Snowden, S. (1997): this volume. 